\title{
Intracranial application of IMRT based radiosurgery to treat multiple or large irregular lesions and verification of infra-red frameless localization system
}

\author{
Joshua D. Lawson · Jia-Zhu Wang · Sameer K. Nath • \\ Roger Rice · Todd Pawlicki · Arno J. Mundt • \\ Kevin Murphy
}

Received: 26 January 2009/Accepted: 9 August 2009/Published online: 20 August 2009

(C) The Author(s) 2009. This article is published with open access at Springerlink.com

\begin{abstract}
We have employed a frameless localization system for intracranial radiosurgery, utilizing a custom biteblock with fiducial markers and an infra-red camera for set-up and monitoring patient position. For multiple brain metastases or large irregular lesions, we use a single-isocenter intensity-modulated approach. We report our quality assurance measurements and our experience using Intensity Modulated Radiosurgery (IMRS) to treat such intracranial lesions. A phantom with integrated targets and fiducial markers was utilized to test the positional accuracy of the system. The frameless localization system was used for patient setup and target localization as well as for motion monitoring during treatment. Inverse optimization planning gave satisfactory dose coverage and critical organ sparing. Patient setup was guided by the infrared camera through fine adjustment in three translational and three rotational degrees for isocenter localization and verified by orthogonal kilovoltage $(\mathrm{kV})$ images, taken before treatment to ensure the accuracy of treatment. The relative localization of the camera based system was verified to be highly accurate along three translational directions of couch motion and couch rotation. After verification, we began treating patients with this technique. About $8-12$ properly selected fixed beams with a single isocenter were sufficient to achieve good dose coverage and organ sparing. Portal dosimetry with an Electronic Portal Imaging Device (EPID) and $\mathrm{kV}$ images provided excellent quality assurance for the IMRS plan and patient setup. The treatment time was less than $60 \mathrm{~min}$ to deliver doses of 16-20 Gy in a single fraction. The camera-
\end{abstract}

J. D. Lawson $(\bowtie) \cdot$ J.-Z. Wang · S. K. Nath · R. Rice .

T. Pawlicki - A. J. Mundt $\cdot$ K. Murphy

Moores Cancer Center, UCSD, 3855 Health Sciences Drive

\#0843, San Diego, CA 92103, USA

e-mail: jlawson@ucsd.edu based system was verified for positional accuracy and was deemed sufficiently accurate for stereotactic treatments. Single isocenter IMRS treatment of multiple brain metastases or large irregular lesions can be done within an acceptable treatment time and gives the benefits of dose-conformity and organ-sparing, easy plan QA, and patient setup verification.

Keywords Frameless · Radiosurgery · Intensity modulated $\cdot$ Infra-red

\section{Introduction}

Radiosurgery has a well-established role in the treatment of intracranial neoplasms both benign and malignant [1-6]. Initially developed by Lars Leksell, the Gamma Knife ${ }^{\circledR}$ remains the oldest treatment method and the gold standard for intracranial radiosurgical treatments [7]. As linear accelerator (linac) technology has advanced, however, linac-based radiosurgery has been increasingly adopted $[8$, 9]. Similar to Gamma Knife ${ }^{\circledR}$ treatment, linac-based radiosurgery has generally been performed using a frame-based system to provide stereotactic guidance.

In order to provide assurance of both patient immobilization and positioning, stereotactic radiosurgery (SRS) has required the patient to have a rigid head-frame affixed to the calvarium. The use of this system provides a 3-dimensional coordinate grid within which any point can be reliably located through the use of the external system alone. This frame is secured using four pins which screw into the calvarium and provide a means for attaching the frame to the treatment table. Patients are generally required to wear this frame for $7-8 \mathrm{~h}$ as imaging and treatment planning is performed just prior to treatment delivery. Treatments usually last 15 min per isocenter with mean 
values for total treatment times on Gamma Knife ${ }^{\circledR}$ Model B, C, and Perfexion recently reported to be 131.0, 129.4, and $114.8 \mathrm{~min}$, respectively [10].

A recently-developed technology allowing frameless intracranial radiosurgery has been described [11-14]. This treatment offers the allure of avoiding the rigid head frame, with the potential negative of diminished positioning accuracy. Clearly, the accuracy of detecting and reporting of fiducial positions by the camera system plays a crucial role in the SRS or Intensity Modulated Radiosurgery (IMRS) procedure [15, 16]. A systematic investigation to quantify the accuracy of the infra-red camera system is therefore of great importance. We describe here the accuracy of such a system, as well as our use of the system for the treatment of multiple intracranial target lesions or large irregularly-shaped lesions [17, 18].

\section{Methods and materials}

The frameless system consists of several components. For each patient, a customized bite-block is made using a dental tray filled with dental cement. After the bite-block is made, a rigid array of four reflective markers is attached to the block. These fiducial markers can be tracked by an infrared camera mounted in the treatment vault (Fig. 1) and are used to determine the planned isocenter position relative to the isocenter of the treatment machine in units of $0.1 \mathrm{~mm}$. After the bite-block has been made, the patient is taken to the treatment vault for verification of reproducibility. In the vault, the patient is fitted with a Reseat Verification Jig, which consists of a head band with a set of reference markers attached. The bite-block is inserted and removed from the patient's mouth ten times, with the optical guidance camera measuring the relative position of the biteblock fiducials and the headband fiducials to determine the repositioning error. Acceptable variation is arbitrarily determined; we have generally accepted $<0.75 \mathrm{~mm}$ for the average of all ten measurements. Patients with large reseat verification error may use a larger margin in the planning target volume (PTV) or switch to intensity modulated radiation treatment (IMRT) utilizing $\mathrm{kV}$ imaging or conebeam computerized tomography (CBCT) for localization.

After reseat verification, the patient proceeds to CT (computed tomography) simulation with the bite-block in position. For better tumor delineation, the CT image set is fused with magnetic resonance (MR) images. Since the immobilization head mask and the bite-block are not used in the MR scan, it can be scheduled before or after CT simulation. The treatment plan is done using a single isocenter and multiple static IMRT beams. The treatment plan, together with the CT images, is sent to the optical guidance computer, where the fiducial locations can be digitized. During treatment delivery, the optical guidance camera tracks the positions of the fiducial markers in real time, and treatment delivery can be manually interrupted by the therapist, if a significant positioning error is observed. We have used a threshold of $0.5-0.75 \mathrm{~mm}$ for treatment interruption and repositioning. Therapists may enter the treatment room to re-adjust the patient position using the optical guidance system.

The frameless system serves two purposes. First, the system can be used for initial patient positioning. The system tracks the positions of the fiducial array for a patient
Fig. 1 A customized bite tray of dental impressions with attached fiducial array (left panel). An infra-red camera mounted on the ceiling to track the position of the optical reflective fiducials (right panel)
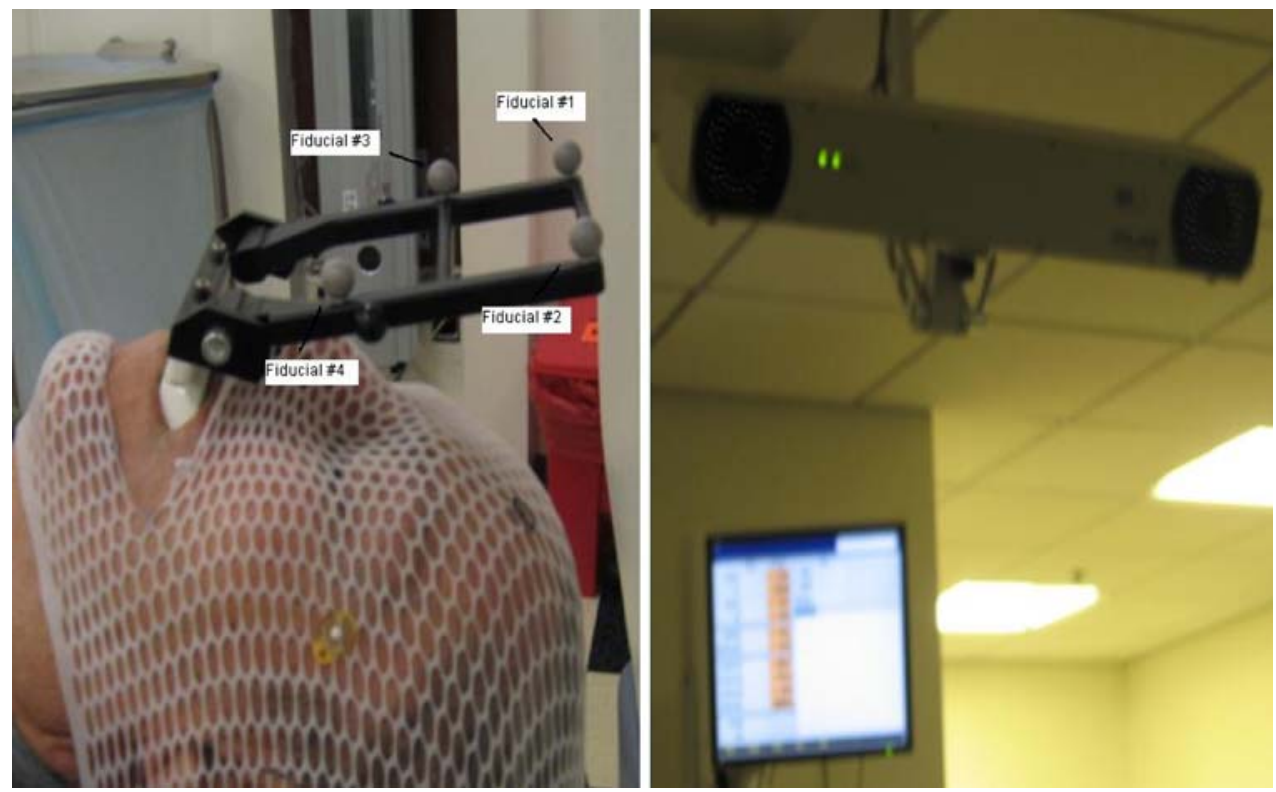
and compares them with the positions localized in the planning CT, as well as pre-calibrated data to determine the target location relative to the isocenter of the treatment machine. Second, the optical guidance camera monitors intrafraction motion by continually assessing the positions of the reflective fiducial markers, allowing treatment interruption in the event of an unacceptable change in patient position.

Initial quality assurance procedures

The phantom experiment of localization accuracy with infra-red optical guidance was carried out by Bova et al. $[18,19]$. Our systematic study on the quality assurance has further verified their early results. A phantom with an integrated target and fiducial markers was mounted either on the treatment couch or on a Brown-Roberts-Wells (BRW) stereotactic floor stand, which enabled positioning with sub-millimeter accuracy near the machine isocenter (Fig. 2). Prior to the phantom study, the camera calibration is first performed using a precise calibration jig that contains reflective markers, which establishes the registration of the optical guidance system with the isocenter of the linac. This procedure is also a prerequisite to each patient treatment.

After making known shifts with the floor stand along the lateral, longitudinal and vertical directions, the reported position from the camera system was recorded. The same procedure was then repeated with the phantom mounted to the couch for greater lateral shifts. Finally, the couch was set at angles based on the camera readings and the digital couch rotations were recorded.

Implementation of patient treatments

After confirming the positional accuracy of the optical guidance system, we began to use the system for radiosurgical treatment of patients. Treatment planning was done using axial images from both a treatment planning MR and CT. Both MR and CT scans were done at $1.25 \mathrm{~mm}$ spacing and a $512 \times 512$ matrix size. A $1.25 \mathrm{~mm}$ interval was selected, because the entire head, including the biteblock, must be included in the imaging field. Although we feel that this will not compromise our accuracy, slight differences may be observed in comparison to studies that use $1.0 \mathrm{~mm}$ spacing. The MR series used was a T1 weighted post-contrast image set; no intravenous or oral contrast was used for acquisition of the CT images. The fusion of MR and CT was carried out in the FastPlan System (Varian Medical Systems, Palo Alto, CA) and the adequacy of the fusion was confirmed visually by the treating neurosurgeon. The fused image sets were exported to the Varian Eclipse Treatment Planning System for IMRS planning.

Target lesions as well as critical organs-at-risk (OAR) were segmented manually. Critical structures included: bilateral eyes and optic nerves, optic chiasm, brainstem, as well as uninvolved brain. The clinical target volume (CTV) consisted of all visualized tumor on either image set. A $2 \mathrm{~mm}$ margin was normally added to create a PTV, and

Fig. 2 A phantom of integrated target and fiducials was mounted either on the treatment couch (right panel), or on a BRW floor stand which can position the phantom near the isocenter with sub-millimeter accuracy (left panel)
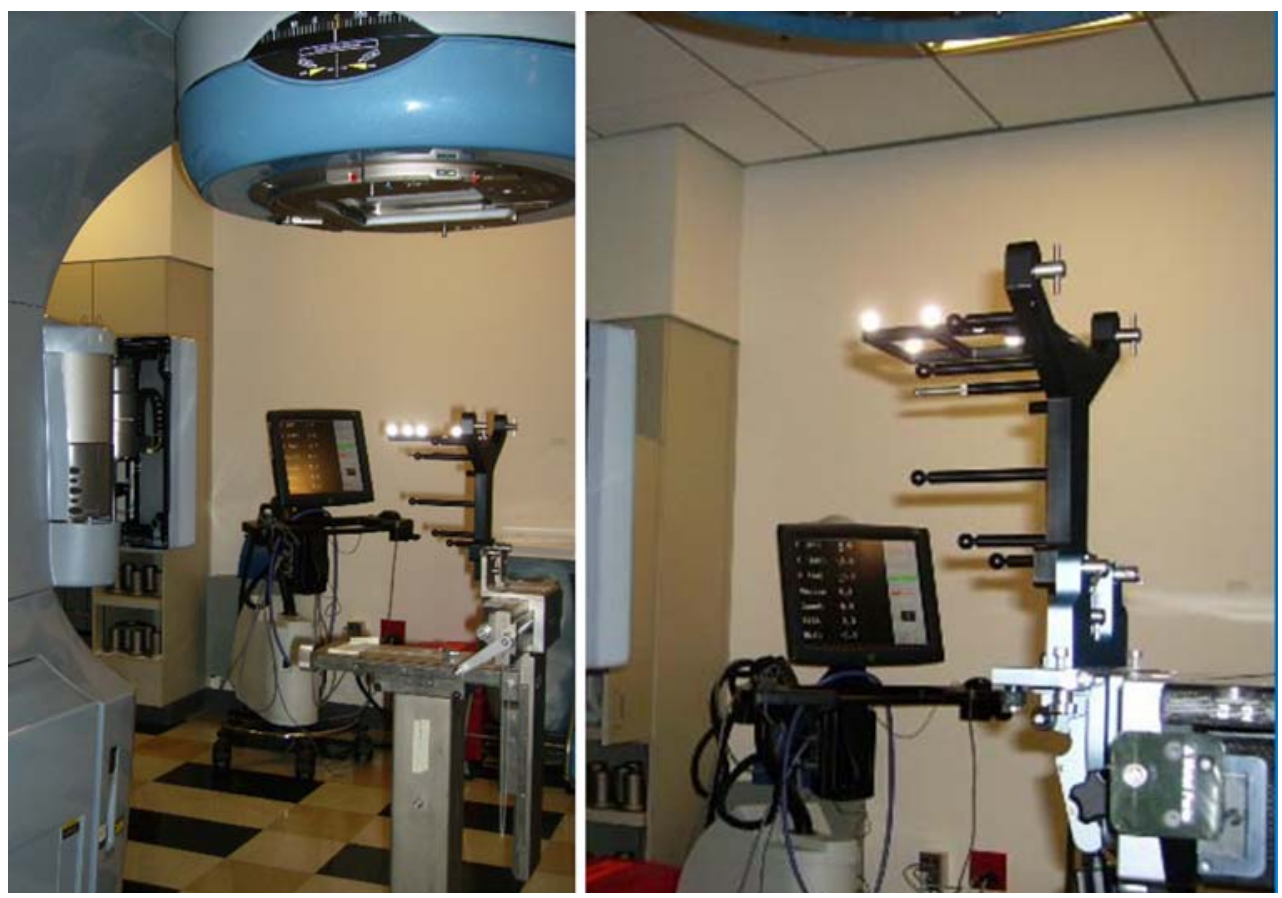
reduced to $1 \mathrm{~mm}$ if close to a critical organ. For patients with a larger reseating verification error $(>0.75 \mathrm{~mm})$, a margin of $3 \mathrm{~mm}$ may be used. A single isocenter was used to treat multiple lesions, with 8-12 carefully selected beams with fixed gantry angles. The number of lesions treated with a single isocenter ranged from 1 to 13 . Prescribed doses were 16-20 Gy with appropriate isodose selection by the treating physician based on the volume: $16 \mathrm{~Gy}$ for large lesions and $20 \mathrm{~Gy}$ for smaller lesions, following the monograph of the Radiosurgery Dose-Volume Relationship similar to what is used in Gamma Knife ${ }^{\circledR}$ treatments. The prescription dose was to the PTV. An isodose line of $88-93 \%$ was normally selected to cover 95$98 \%$ of the PTV. The gantry angle and couch rotation of each beam was carefully selected and evaluated in the beam's eye view (BEV) to provide optimal organ sparing and target coverage. All the beams were designed to have minimal overlap with each other and, as a whole, covered a solid angle as large as possible.

The sliding-window technique was used in all the IMRS delivery, with a selected dose rate of $1000 \mathrm{MU} / \mathrm{min}$; maximum MU per beam is 1999 and maximum field size (jaw setting) is $15 \mathrm{~cm} \times 15 \mathrm{~cm}$. It was found that with proper gantry and couch orientations, the field size of $15 \mathrm{~cm} \times 15 \mathrm{~cm}$ could cover almost all of the lesions. In case a lesion was not covered properly by one beam, it could still be covered by other beams at different orientations. For patient-related IMRT quality assurance, the planar dose distribution was verified for each beam using portal dosimetry on an as 1000 EPID detector prior to patient treatment $[19,20]$.

In treatment planning, two orthogonal beams with digitally reconstructed radiographs (DRR) were created in the IMRS plan. At the time of patient treatment, the optical guidance system was used for initial patient positioning. Additionally, a pair of orthogonal $\mathrm{kV}$ images was acquired using On-Board Imaging (OBI) prior to treatment. These were compared to the DRRs from treatment planning for further validation of patient setup (Fig. 3). The use of these images allows detection of a misplaced or unseated biteblock which might not otherwise be detected and could lead to inaccurate localization of the target and mistreatment.

\section{Results}

The results of our initial quality assurance investigation are shown in Fig. 4. The relationship between the actual known directional shifts and the measured shifts from the optical guidance camera readings is shown in each curve. The four curves represent lateral, longitudinal, vertical and rotational shifts. As the test target of the phantom is positioned at the linac isocenter, the camera readings are all at zero values, while the floor stand has lateral position at $0.0 \mathrm{~cm}$ and the digital couch position at lateral $996.2 \mathrm{~cm}$, longitudinal $66.9 \mathrm{~cm}$, vertical $22.1 \mathrm{~cm}$, and rotation at $0.0^{\circ}$ angle. The floor stand readings with a resolution of $0.1 \mathrm{~mm}$ are reported along the lateral direction only from 0 to $1 \mathrm{~cm}$. The resolution of the couch digital readings are $1 \mathrm{~mm}$ along all three directions, and $0.1^{\circ}$ in couch rotation.

In order to combine the results from the floor stand and the couch mount, the lateral home position of the couch is re-set to $0.0 \mathrm{~cm}$, and the right and left shifts given either by floor stand or by couch, as well as the camera readings, are all converted to positive numbers and plotted together in Fig. 4a. The correlation of lateral shifts of camera readings and actual known shifts yields a perfect match of a straight line in Fig. $4 \mathrm{a}$, of a slope $=1$ with $R^{2}=1$. The same approach is applied to the longitudinal shifts in Fig. $4 \mathrm{~b}$ and rotational in Fig. 4d. The vertical couch home position is kept unchanged at $22.1 \mathrm{~cm}$ such that the up and down shifts are separated in Fig. 4c. In each measurement, the correlation results in a straight line of a slope $=1$ with $R^{2}=1$. The resolution of the camera readings is $0.1 \mathrm{~mm}$ in all three translational directions and 0.1 degree in rotational axes. The excellent agreement of the camera system and the actual fiducial positions confirmed the accuracy of the optical guided frameless positioning system. The utilization of portal dosimetry and $\mathrm{kV}$ images as verification provided further quality assurance for the IMRS plan and patient setup.

After verification of accuracy, we began treating patients with this technique. Two treatment plans for patients treated with our single-isocenter IMRS technique are shown in Fig. 5 as representative cases. One patient has three distinct lesions, including one of an irregular shape (top panel), with a combined CTV of $14.46 \mathrm{cc}$. Each lesion was segmented separately, and the PTV consists of all three lesions together with a $2 \mathrm{~mm}$ margin, resulting in a total volume of $23.98 \mathrm{cc}$. $16 \mathrm{~Gy}$ was prescribed to the combined PTV. The mean dose to normal brain and brainstem were 3.2 and $0.6 \mathrm{~Gy}$, respectively. Eyes, optic nerves, and chiasm all received minimal dose $(<0.01 \mathrm{~Gy})$. The top right pane shows the excellent tumor coverage and relative sparing of OAR achieved with a single-isocenter plan. The bottom two panes show the plan for a patient treated to 12 lesions. Again these were contoured separately and together formed the PTV with a total volume of $15.98 \mathrm{cc}$, where a $3 \mathrm{~mm}$ margin was added to the original CTV of $3.8 \mathrm{cc}$. $16 \mathrm{cGy}$ was prescribed to the combined PTV for this patient. In addition, at the bottom right is shown the portal dosimetry done for quality assurance on the IMRS plan using the Electronic Portal Imaging Device (EPID). 
A

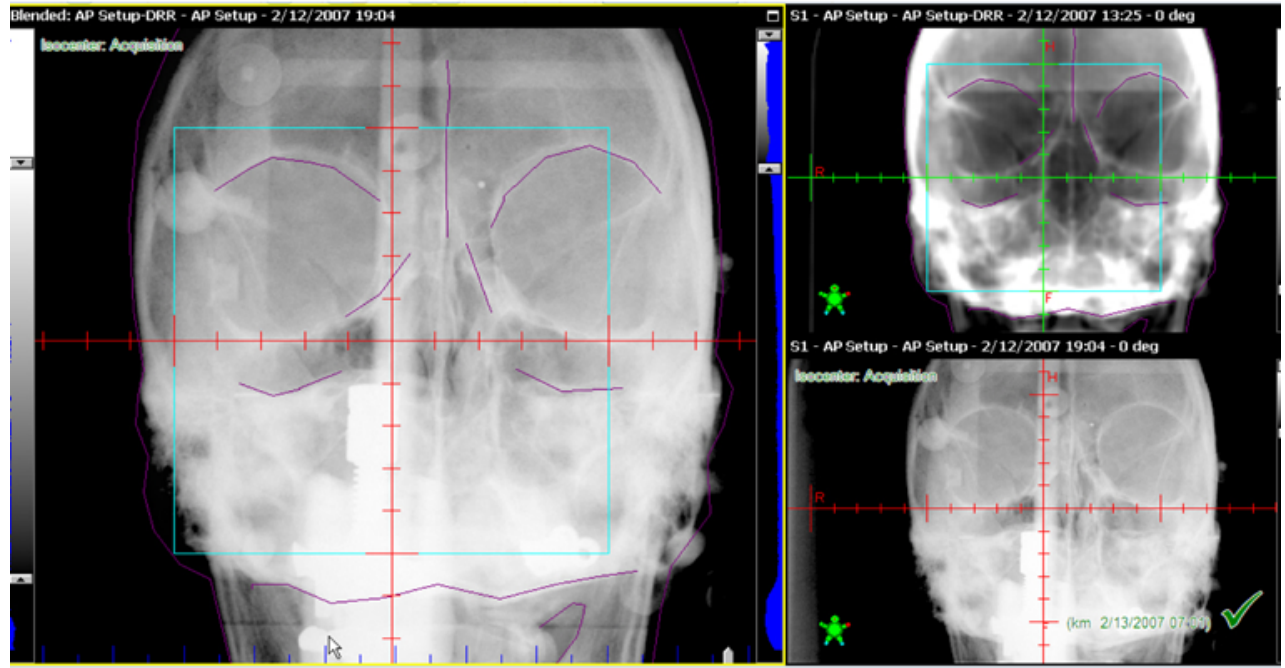

B

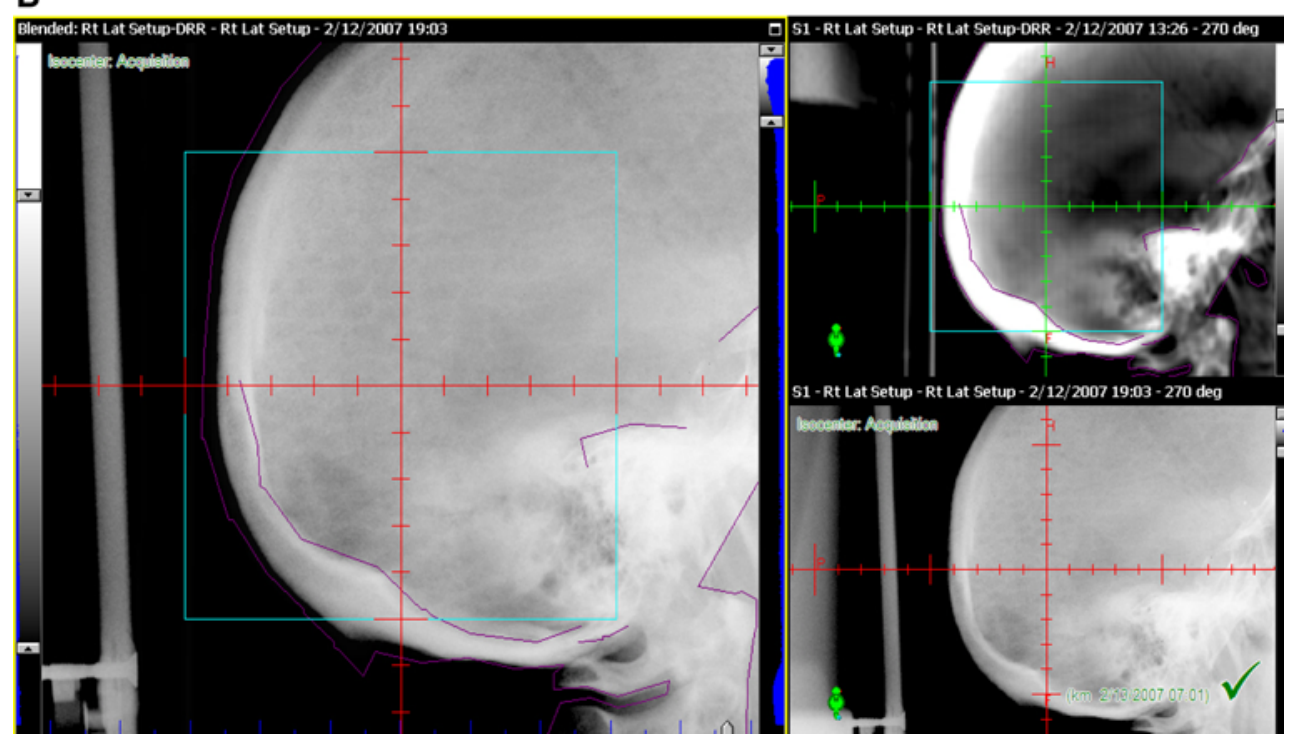

Fig. 3 AP (a) and lateral (b) DRR, setup images, and blended images used for setup verification at the time of treatment delivery

\section{Discussion}

Stereotactic guidance provides a reliable and reproducible surrogate through which any point can be accurately localized. The use of a rigid head frame maintains stability in positioning, preventing patient motion during treatment delivery. This system allows for confidence in the accurate delivery of very high doses to target lesions with very small expansions for setup uncertainty [21]. However, many patients are resistant to the head frame, and there is morbidity associated with its use. Frameless radiosurgery can offer the patient the advantages of high-dose, single-fraction treatment without requiring the head frame. Reported experience with frameless radiosurgery remains limited. The largest series to date includes 64 patients, most of whom also received surgical resection, whole brain radiation treatment, or both. In this series, overall local control was similar to that reported for frame-based radiosurgery at $88 \%$ [14, 22-24]. Treatment plans on this frameless series employed 1-7 isocenters and 5-35 treatment arcs.

Instead of a multiple-isocenter approach, we have used a single-isocenter, intensity modulated approach for the treatment of patients with multiple intracranial targets or large, irregularly shaped targets. In all cases a single treatment fraction was able to deliver adequate target coverage and sparing of nearby critical normal structures. In a previous analysis of ten patients treated with our single-isocenter technique, the median maximal and minimal PTV coverage was found to be 107 and $87 \%$, respectively, with a median integral dose to normal brain 
A
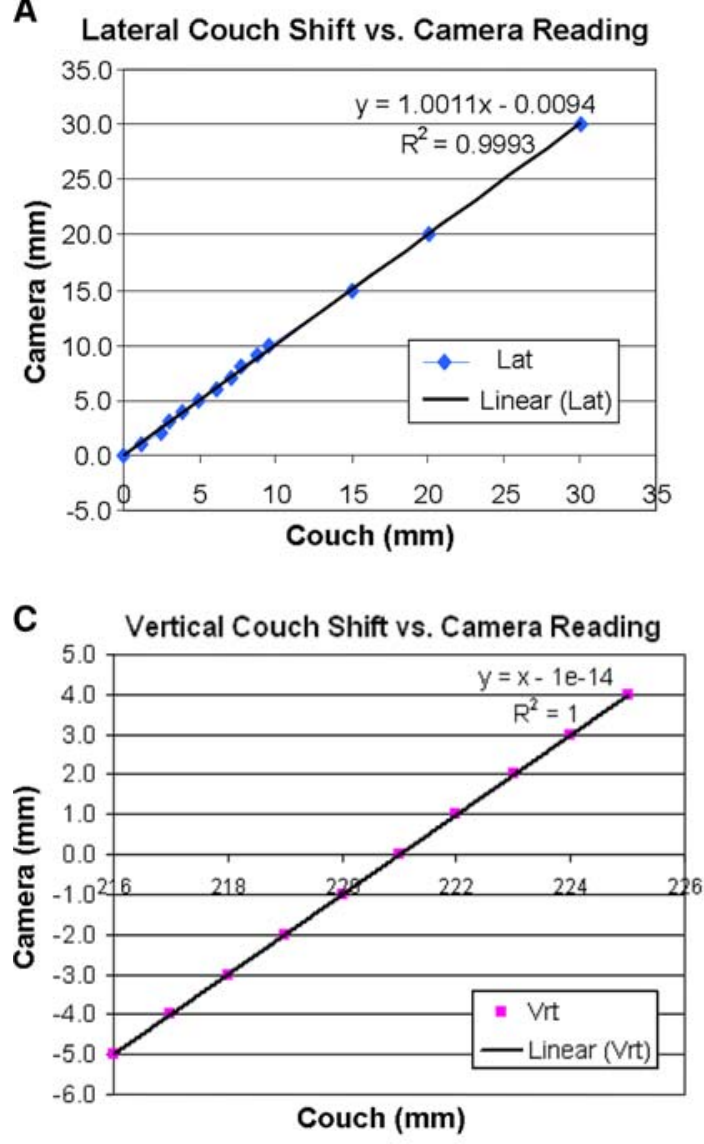

Fig. 4 Correlation of measurement of lateral, longitudinal, and vertical $(\mathbf{a}-\mathbf{c})$ shifts between camera readings and actual shifts given either by a floor stand (lateral only) or couch digital readout (all directions). The home couch positions are re-set to 0 and shifts in either direction of axis are combined in the graphs of $\mathbf{a}$ and $\mathbf{b}$. In $\mathbf{c}$, the vertical home position of $221 \mathrm{~mm}$ is kept unchanged and up and

of 6.2 Gy [25]. Additionally, in a side-by-side comparison of a patient planned using both a single- and multipleisocenter approach, median integral dose to brain was 4.5 and 1.6 Gy for a single- versus multiple-isocenter plan, respectively. Toxicity was found to be generally mild and acceptable. These previous results in combination with our current quality assurance analysis suggest that this technique may offer a more streamlined approach to treatment planning without compromising the quality of treatment.

As a comparison, the hot spot inside the PTV of an IMRS plan is typically $15-20 \%$ greater than the prescribed dose, whereas the dose to the center of each lesion is twice greater than the prescribed dose in Gamma Knife $^{\circledR}$ and $20 \%$ or higher in cone-based arc linac plans. On the other hand, the dose fall-off outside PTV in IMRS plans is less rapid than that in Gamma Knife ${ }^{\circledR}$ or in a cone based arc plan, causing the integral dose to normal brain to be slightly higher with IMRS. These observations
B Longitudinal Couch Shift vs. Camera Reading
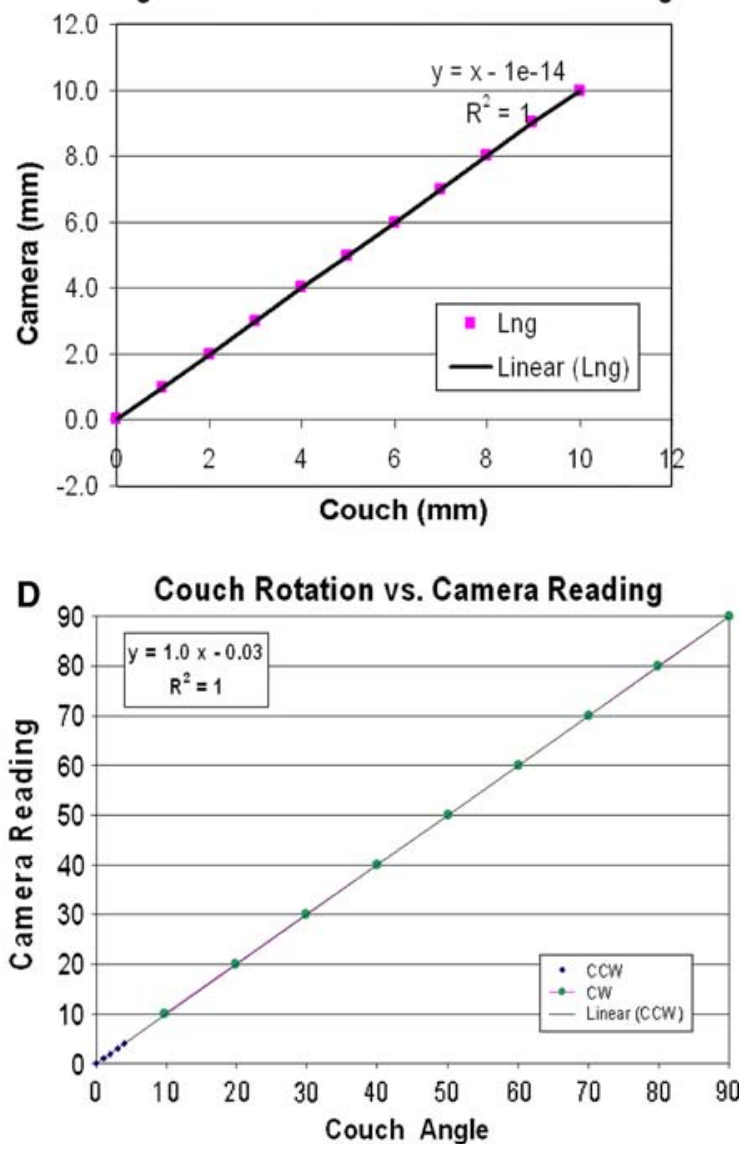

down shifts are not combined. The clockwise $(\mathrm{CW})$ and counterclockwise rotation of the couch are again combined in $\mathbf{d}$. CCW rotation were carried out in increment of $1^{\circ}$ each till $5^{\circ}$, and then changed to every $10^{\circ}$, and up to $90^{\circ}$, while the $\mathrm{CW}$ rotation was done in every $10^{\circ}$

are better demonstrated in dose-volume histograms (DVH) from PTV and normal brain for IMRS plans, such as those shown in Fig. 5. Although integral dose to normal brain was increased, the clinical significance of this elevation is unclear. Treatment with single-isocenter IMRS was well-tolerated, with minimal acute toxicity reported by our patients in this series. Long-term assessment of post-IMRS patients, including an analysis of local control, toxicity, and survival outcomes, is currently underway and will help to define the true benefits and risks of this technique.

The use of a frameless technique offers additional patient convenience. Many patients are resistant to the head frame, and find it difficult to wear for the number of hours required. Frameless techniques not only alleviate this problem for single-fraction treatments, but also offer the option of hypofractionated treatment or single-fraction treatment done on a separate day from treatment planning [26]. In addition, the total treatment time for patient setup 

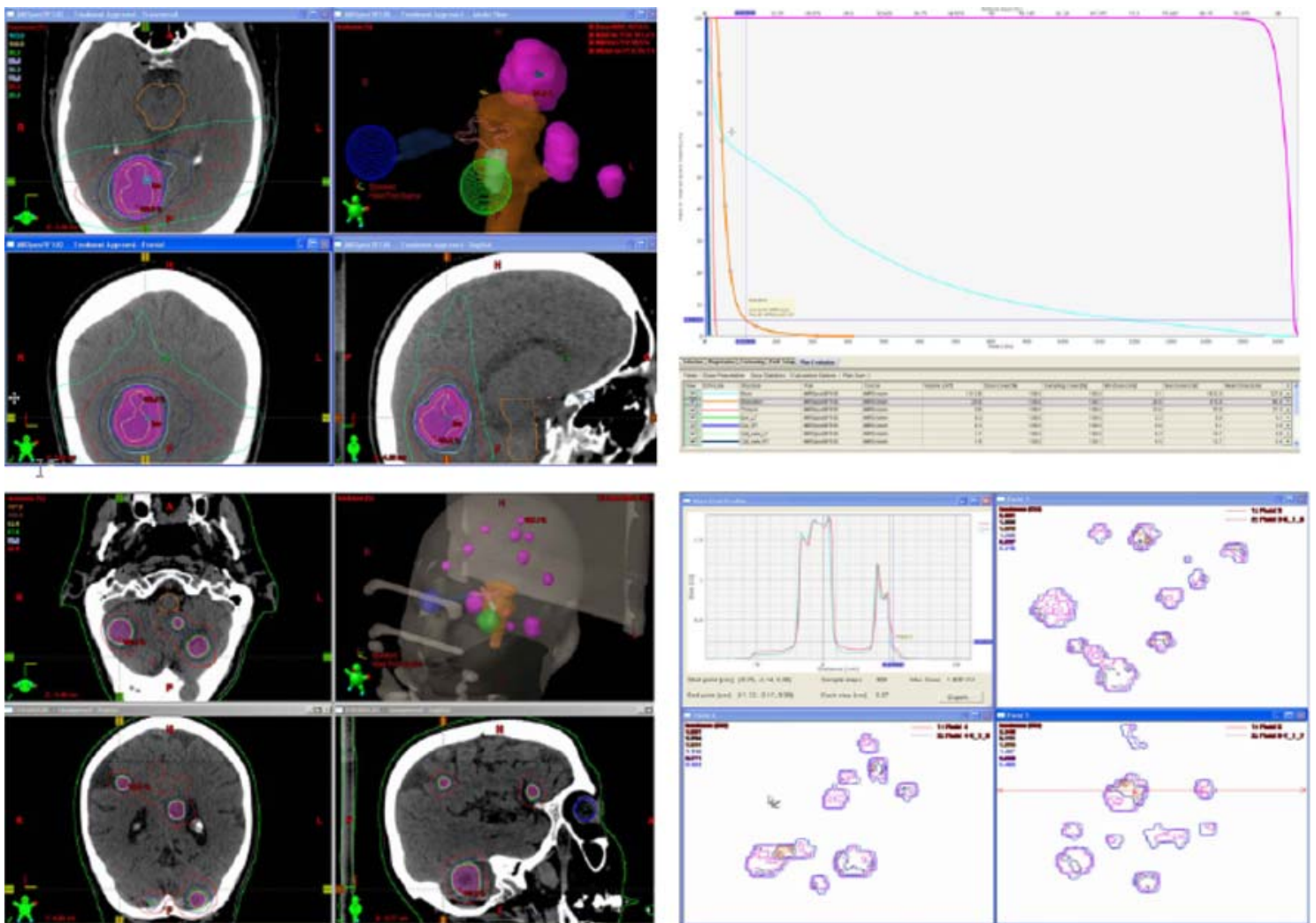

Fig. 5 The IMRS plan of a patient with three lesions (top). 16 Gy was prescribed to the combined PTV. The normal brain and brainstem are shown in light blue and orange. At bottom is the IMRS plan of a patient with 12 lesions. The quality assurance of IMRS plans was done by portal dosimetry as shown in the lower right. The solid lines

represent the calculated planar dose distributions and the dotted lines the measured dose using EPID. The horizontal profile of such planar dose distribution of one beam is displayed, where the superimposed curves represent the calculated and measured dose profiles (Color figure online)

validation and dose delivery was about $45 \mathrm{~min}$ to deliver doses of 16-20 Gy in each case. Patients with larger PTV can be treated with multiple fractions, typically of a prescription dose 6 Gy per fraction, a total of $30 \mathrm{~Gy}$ delivered in five fractions, which is made possible only by the use of frameless localization system.

Our quality assurance procedures have shown the optical guidance system to be accurate and reliable; a further advantage of its use is the ability for intrafraction monitoring of patient position. The system allows for treatment interruption if the measured displacement of the fiducial markers is outside the predetermined tolerances.

The patient setup, after target localization through the optical guidance camera system, is verified by the orthogonal KV-image pair using OBI and comparing with the DRR images. KV image-DRR comparison serves as a verification tool to ensure patient positioning accuracy prior to the radiation treatment and is not routinely used as a patient repositioning tool in the IMRS treatment.

In some cases, due to issues with bite-block reseating, where isocenter misalignment is identified as $2 \mathrm{~mm}$ or greater when matching $\mathrm{KV}$ images with DRRs, patient

treatment may proceed as IMRT treatment without utilizing the optical guidance system. This is a clinical decision, made after images have been reviewed by therapist, physicist, and physician together, and the patient is re-positioned based on OBI to make isocenter shift prior to the radiation treatment.

For patients with dentures, we have found that with the help of denture adhesive treatment can proceed with CT simulation and keep the reproducibility at treatment. This frameless immobilization may not be suitable to those who can not hold the bite block in place. For those patients, IMRT treatment may be a better approach.

\section{Conclusion}

Ours is a novel strategy-frameless radiosurgical simultaneous treatment of multiple intracranial targets with a single-isocenter intensity modulated plan. We believe this is an appealing alternative to conventional frame-based systems, offering both convenience and comfort to patients while maintaining overall plan quality. 
Open Access This article is distributed under the terms of the Creative Commons Attribution Noncommercial License which permits any noncommercial use, distribution, and reproduction in any medium, provided the original author(s) and source are credited.

\section{References}

1. Flickinger JC et al (1994) A multi-institutional experience with stereotactic radiosurgery for solitary brain metastasis. Int J Radiat Oncol Biol Phys 28(4):797-802

2. Alexander E III et al (1995) Stereotactic radiosurgery for the definitive, noninvasive treatment of brain metastases. J Natl Cancer Inst 87(1):34-40. doi:10.1093/jnci/87.1.34

3. Shaw E et al (1996) Radiosurgery for the treatment of previously irradiated recurrent primary brain tumors and brain metastases: initial report of radiation therapy oncology group protocol (9005). Int J Radiat Oncol Biol Phys 34(3):647-654. doi:10.1016/ 0360-3016(95)02106-X

4. Yoon SC et al (1998) Clinical results of 24 pituitary macroadenomas with linac-based stereotactic radiosurgery. Int $\mathrm{J}$ Radiat Oncol Biol Phys 41(4):849-853. doi:10.1016/S0360-3016(98) 00124-2

5. Maruyama $\mathrm{K}$ et al (2005) The risk of hemorrhage after radiosurgery for cerebral arteriovenous malformations. N Engl J Med 352(2):146-153. doi:10.1056/NEJMoa040907

6. Poznanovic SA, Cass SP, Kavanagh BD (2006) Short-term tumor control and acute toxicity after stereotactic radiosurgery for glomus jugulare tumors. Otolaryngol Head Neck Surg 134(3): 437-442. doi:10.1016/j.otohns.2005.10.040

7. Larsson B et al (1958) The high-energy proton beam as a neurosurgical tool. Nature 182(4644):1222-1223. doi:10.1038/1821 $222 \mathrm{a} 0$

8. Hitchcock E et al (1989) Stereotactic LINAC radiosurgery. Br J Neurosurg 3(3):305-312. doi:10.3109/02688698909002808

9. Alexander E III, Loeffler JS (1992) Radiosurgery using a modified linear accelerator. Neurosurg Clin N Am 3(1):167-190

10. Lindquist C, Paddick I (2007) The Leksell Gamma Knife Perfexion and comparisons with its predecessors. Neurosurgery 61(3)(Suppl):130-140 (discussion 140-141). doi:10.1227/01.neu. 0000289726.35330.8a

11. Bova FJ et al (1997) The University of Florida frameless highprecision stereotactic radiotherapy system. Int $\mathrm{J}$ Radiat Oncol Biol Phys 38(4):875-882. doi:10.1016/S0360-3016(97)00055-2

12. Buatti JM et al (1998) Preliminary experience with frameless stereotactic radiotherapy. Int J Radiat Oncol Biol Phys 42(3): 591-599. doi:10.1016/S0360-3016(98)00276-4
13. Ryken TC et al (2001) Initial clinical experience with frameless stereotactic radiosurgery: analysis of accuracy and feasibility. Int J Radiat Oncol Biol Phys 51(4):1152-1158. doi:10.1016/ S0360-3016(01)01756-4

14. Kamath $\mathrm{R}$ et al (2005) Initial clinical experience with frameless radiosurgery for patients with intracranial metastases. Int J Radiat Oncol Biol Phys 61(5):1467-1472. doi:10.1016/j.ijrobp.2004. 08.021

15. Kim KH et al (2003) Isocenter accuracy in frameless stereotactic radiotherapy using implanted fiducials. Int J Radiat Oncol Biol Phys 56(1):266-273. doi:10.1016/S0360-3016(03)00088-9

16. Murphy MJ et al (2003) Patterns of patient movement during frameless image-guided radiosurgery. Int $\mathrm{J}$ Radiat Oncol Biol Phys 55(5):1400-1408. doi:10.1016/S0360-3016(02)04597-2

17. Wang J, Rice R (2007) SU-FF-T-448: verification of infra-red camera localization system for stereotactic external beam treatment. Med Phys 34(6):2504-2505

18. Wang $\mathbf{J}$ et al (2007) SU-FF-T-269: intracranial applications of IMRT based stereotactic radiosurgery to treat multiple or large irregular lesions. Med Phys 34(6):2463. doi:10.1118/1.2760930

19. Ting J (2005) Commissioning and dosimetric quality assurance. In A Mundt, JC Roeske (eds) Intensity modulated radiation therapy, a clinical perspective. Decker Inc., Hamilton

20. Wang J, Rice R, Marcus L (2006) MO-D-224A-02: clinical experience in using EPID for quantitative verification of IMRT dose distributions. Med Phys 33(6):2167. doi:10.1118/1.2241437

21. Noel G et al (2003) Radiosurgery for brain metastasis: impact of CTV on local control. Radiother Oncol 68(1):15-21. doi:10.1016/ S0167-8140(03)00207-X

22. Pirzkall A et al (1998) Radiosurgery alone or in combination with whole-brain radiotherapy for brain metastases. J Clin Oncol 16(11):3563-3569

23. Shirato $\mathrm{H}$ et al (1997) Stereotactic irradiation without wholebrain irradiation for single brain metastasis. Int J Radiat Oncol Biol Phys 37(2):385-391. doi:10.1016/S0360-3016(96)00488-9

24. Sneed PK et al (2002) A multi-institutional review of radiosurgery alone vs. radiosurgery with whole brain radiotherapy as the initial management of brain metastases. Int J Radiat Oncol Biol Phys 53(3):519-526. doi:10.1016/S0360-3016(02)02770-0

25. Wang $\mathbf{J}$ et al (2007) Intracranial applications of IMRT based stereotactic radiosurgery to treat multiple or large irregular lesions. Med Phys 34(6):2463. doi:10.1118/1.2760930

26. Narayana A et al (2007) Hypofractionated stereotactic radiotherapy using intensity-modulated radiotherapy in patients with one or two brain metastases. Stereotact Funct Neurosurg 85(23):82-87. doi:10.1159/000097923 